\title{
Oligomer-Targeting Prevention of Neurodegenerative Dementia by Intranasal Rifampicin and Resveratrol Combination - A Preclinical Study in Model Mice
}

\section{OPEN ACCESS}

Edited by:

Sergio T. Ferreira,

Federal University of Rio de Janeiro,

Brazil

Reviewed by:

Wieslawa Agnieszka Fogel, Medical University of Łódź, Poland

Alain Buisson,

Université Grenoble Alpes, France

Sylvain Lesné,

University of Minnesota Twin Cities,

United States

*Correspondence:

Takami Tomiyama

tomi@med.osaka-cu.ac.jp

Specialty section:

This article was submitted to

Neuropharmacology,

a section of the journal

Frontiers in Neuroscience

Received: 24 August 2021 Accepted: 23 November 2021 Published: 13 December 2021

Citation:

Umeda T, Sakai A, Shigemori K, Yokota A, Kumagai T and Tomiyama $T$ (2021) Oligomer-Targeting Prevention

of Neurodegenerative Dementia by Intranasal Rifampicin and Resveratrol Combination - A Preclinical Study

in Model Mice.

Front. Neurosci. 15:763476. doi: 10.3389/fnins.2021.763476

\section{Tomohiro Umeda ${ }^{1}$, Ayumi Sakai ${ }^{1}$, Keiko Shigemori ${ }^{1}$, Ayumi Yokota1 , Toru Kumagai ${ }^{2}$ and Takami Tomiyama1,2*}

${ }^{1}$ Department of Translational Neuroscience, Osaka City University Graduate School of Medicine, Osaka, Japan, ${ }^{2}$ Medilabo RFP, Inc., Kyoto, Japan

Amyloidogenic protein oligomers are thought to play an important role in the pathogenesis of neurodegenerative dementia, including Alzheimer's disease, frontotemporal dementia, and dementia with Lewy bodies. Previously we demonstrated that oral or intranasal rifampicin improved the cognition of APP-, tau-, and $\alpha$-synucleintransgenic mice by reducing the amount of $A \beta$, tau, and $\alpha$-synuclein oligomers in the brain. In the present study, to explore more effective and safer medications for dementia, we tested the drug combination of rifampicin and resveratrol, which is a multifunctional natural polyphenol with the potential to antagonize the adverse effects of rifampicin. The mixture was intranasally administered to APP-, tau-, and $\alpha$-synucleintransgenic mice, and their memory and oligomer-related pathologies were evaluated. Compared with rifampicin and resveratrol alone, the combinatorial medicine significantly improved mouse cognition, reduced amyloid oligomer accumulation, and recovered synaptophysin levels in the hippocampus. The plasma levels of liver enzymes, which reflect hepatic injury and normally increase by rifampicin treatment, remained normal by the combination treatment. Notably, resveratrol alone and the combinatorial medicine, but not rifampicin alone, enhanced the levels of brain-derived neurotrophic factor (BDNF) and its precursor, pro-BDNF, in the hippocampus. Furthermore, the combination showed a synergistic effect in ameliorating mouse cognition. These results show the advantages of this combinatorial medicine with regards to safety and effectiveness over single-drug rifampicin. Our findings may provide a feasible means for the prevention of neurodegenerative dementia that targets toxic oligomers.

Keywords: rifampicin, resveratrol, intranasal administration, $A \beta$, tau, $\alpha$-synuclein, oligomer, prevention

\section{INTRODUCTION}

Neurodegenerative dementia is defined as neurodegenerative diseases with a main clinical symptom of dementia, which includes Alzheimer's disease (AD), frontotemporal dementia (FTD), and dementia with Lewy bodies (DLB). Parkinson's disease (PD) is also known to pose dementia when its pathologies spread into the cerebral cortex. These disorders are characterized by the cerebral 
accumulation of disease-specific amyloidogenic proteins: $A \beta$ and tau in $\mathrm{AD}$, tau or TDP-43 in FTD, and $\alpha$-synuclein in DLB and $\mathrm{PD}$. These proteins tend to self-aggregate into insoluble fibrils with the $\beta$-sheet structure leading to the formation of characteristic pathological inclusions in the brain, such as senile plaques composed of $A \beta$, neurofibrillary tangles of hyperphosphorylated tau, and Lewy bodies of phosphorylated $\alpha$-synuclein. While these inclusions are helpful for the differential diagnosis of neurodegenerative diseases, accumulating evidence indicates that the real causal culprit of the disease is smaller, soluble oligomers of the proteins. For example, synaptic dysfunction, which is assumed an immediate early symptom in $\mathrm{AD}$, is caused by soluble $\mathrm{A} \beta$ oligomers (Cline et al., 2018; Li and Selkoe, 2020), and A $\beta$ oligomers trigger the pathological cascade of AD including tau hyperphosphorylation, glial activation, and neuronal loss (Tomiyama et al., 2010; Cline et al., 2018). Tau oligomers (Maeda and Takashima, 2019; Hill et al., 2020) and $\alpha$-synuclein oligomers (Bengoa-Vergniory et al., 2017; Kayed et al., 2020) are also suggested to play a crucial role in the pathogenesis of tauopathy and $\alpha$-synucleinopathy, respectively.

Despite the vigorous efforts of researchers and pharmaceutical companies, there is no effective cure for neurodegenerative dementia. Many drug candidates have been developed, but most have failed to show beneficial effects on patients' cognition in clinical trials. These failures are primarily attributed to two main reasons: the late timing of medication and the wrong drug target. The treatment should be started early, before the neurodegeneration proceeds, and the drug target should be set to the toxic oligomers. We previously demonstrated that a wellknown antibiotic, rifampicin, inhibited the oligomerization of $\mathrm{A} \beta$, tau, and $\alpha$-synuclein in vitro and that the activity was specific to pathological amyloidogenic proteins but not to physiologically assembling proteins (Umeda et al., 2016). Furthermore, when orally administered to APP- and tau-transgenic (Tg) mice, rifampicin reduced $A \beta$ and tau oligomers in the brain and improved the cognition of the mice (Umeda et al., 2016). These results suggest that rifampicin is a promising oligomer-targeting medicine and can prevent neurodegenerative dementia when administered early before the neurodegeneration.

However, oral rifampicin occasionally induces adverse effects such as liver dysfunction and drug-drug interaction (Drugs.com website $\left.^{1}\right)$. Such adverse events are considered to occur during the first pass of rifampicin from the intestine to the liver. To avoid this pathway, we sought a different administration route. We noted that the drug injected into the nasal cavity is efficiently delivered to the brain by passing through the nasal mucosa epithelium and transported less to the liver (Erdõ et al., 2018). Thus, we compared three administration routes: oral, intranasal, and subcutaneous, on the safety, brain delivery, and therapeutic efficacy in APP-Tg mice (Umeda et al., 2018). Intranasal administration showed the highest brain delivery and therapeutic efficacy and improved safety. Taking the treatment invasiveness into consideration, we concluded that intranasal administration was the best way for long-term rifampicin treatment. Thereafter, we expanded our study to $\alpha$-synuclein-Tg mice to show that nasal

${ }^{1}$ https://www.drugs.com/mtm/rifampin.html rifampicin reduced brain $\alpha$-synuclein oligomers and improved mouse cognition (Umeda et al., 2021).

In the prevention of neurodegenerative dementia, drug treatment is continued for a long period, and therefore, preventive medicines must be extremely safe. To further secure the safety of nasal rifampicin, we hypothesized that rifampicin's undesired actions could be antagonized by other compounds. Thus, we explored the literature for a compound that possesses hepatoprotective actions opposite to rifampicin and, if possible, additional clinical effects that rifampicin does not show. We consequently selected trans-resveratrol as a candidate. This compound is a stilbene-type natural polyphenol and a phytochemical highly contained in grapes and berries. Resveratrol is a multifunctional agent that shows antioxidant, anti-inflammatory, anti-diabetes, anti-cancer, and neuroprotective effects (Meng et al., 2020; Rahman et al., 2020). It shows high tolerability and low toxicity in humans and is widely used as a dietary supplement. Furthermore, transresveratrol inhibits the expression of cytochrome P450 3A4 (CYP3A4) and P-glycoprotein (Deng et al., 2014; El-Readi et al., 2019), both of which are enhanced by rifampicin (Kim et al., 2008; Tan et al., 2016), implying that resveratrol can neutralize the hepatotoxicity and drug-drug interaction of rifampicin. In addition, resveratrol increases the expression of brain-derived neurotrophic factor (BDNF) in animals (Shen et al., 2018; Chen et al., 2021), an effect not seen with rifampicin. BDNF plays an important role in synaptic plasticity and memory, and its levels are reduced in the brains of patients with neurodegenerative and psychiatric disorders (Miranda et al., 2019). However, resveratrol has a pharmacokinetic problem: due to its extensive glucuronidation and sulfation in the intestine and liver, the bioavailability of oral resveratrol is very low (Cottart et al., 2014). Thus, in the present study, we tested the intranasal combination of rifampicin and resveratrol in multiple mouse models of neurodegenerative dementia. The results show several advantages of this combinatorial medicine in terms of safety and effectiveness over single-drug rifampicin.

\section{MATERIALS AND METHODS}

\section{Mice}

Four kinds of Tg mice were used. APP OsK and tau784 mice were generated in our laboratory, APP23 mice were kindly provided by Novartis Pharma, Inc., and Hua-Syn (A53T) line G23 mice, simply referred to as $\alpha$ Syn-Tg mice here, were purchased from the Jackson Laboratory (Bar Harbor, ME, United States). APP OSK mice express human APP695 with the Osaka (E693 $\Delta$ ) mutation under the mouse prion protein promoter and show an intraneuronal accumulation of $\mathrm{A} \beta$ oligomers and memory impairment at 8 months without forming amyloid plaques (Tomiyama et al., 2010). APP23 mice express human APP751 with the Swedish (KM670/671NL) mutation under the mouse Thy-1 promoter (Sturchler-Pierrat et al., 1997) and exhibit cognitive decline at 3 months and amyloid plaques at 6 months (Van Dam et al., 2003). Tau784 mice express both wild-type 3repeat and 4-repeat human tau (tau410 and tau441) under the 
mouse CaMKIIa promoter with a dominant expression of 4repeat human tau at adult ages by the presence of tau intron $10+16 \mathrm{C}-\mathrm{T}$ mutation in the transgene (Umeda et al., 2013). They develop tau hyperphosphorylation, tau oligomer accumulation, and cognitive dysfunction at 6 months and neurofibrillary tangles at 15 months (Umeda et al., 2015). aSyn-Tg mice express A53Tmutant human $\alpha$-synuclein under the mouse prion protein promoter (Lee et al., 2002) and display $\alpha$-synuclein oligomer accumulation and cognitive impairment at 6 months and motor dysfunction at 9 months (Umeda et al., 2021). All Tg mice were maintained and used as heterozygotes.

\section{Rifampicin and Resveratrol Treatment}

Administration of rifampicin and resveratrol to mice was started at an age at which oligomer pathology and cognitive dysfunction progressed enough. We assume that the pathological stage in model mice at the age we chose corresponds to the preclinical to prodromal stage of neurodegenerative dementia in humans. APP $P_{\text {OSK }}$ mice 13-months old were divided into 5 groups containing 5-6 male and 4-6 female each based on the drug treatment: rifampicin alone $(0.02 \mathrm{mg} /$ day $)$, low-dose resveratrol alone (0.02 mg/day), high-dose resveratrol alone $(0.1 \mathrm{mg} /$ day $)$, combinatorial medicine $(0.02 \mathrm{mg}$ rifampicin $+0.02 \mathrm{mg}$ resveratrol/day), and carboxymethylcellulose (CMC) as a control. APP23 mice at 16-17-months old were divided into 2 groups containing 5-6 male and 3-4 female each: one for combinatorial medicine (0.02 $\mathrm{mg}$ each drug/day) and the other for CMC. Tau784 mice 13-14-months old were also divided into 5 groups containing 5-7 male and 3-5 female each: rifampicin alone (0.02 mg/day), resveratrol alone (0.02 mg/day), lowdose combinatorial medicine $(0.01 \mathrm{mg}$ each drug/day), highdose combinatorial medicine (0.02 mg each drug/day), and CMC. aSyn-Tg mice 6-months old were divided into 3 groups containing 3-4 male and 5-8 female each: low-dose combinatorial medicine ( $0.01 \mathrm{mg}$ each drug/day), high-dose combinatorial medicine ( $0.02 \mathrm{mg}$ each drug/day), and CMC. Rifampicin (Sigma-Aldrich, St. Luis, MO, United States) and trans-resveratrol (Sigma-Aldrich) were dissolved to 2 or $4 \mathrm{mg} / \mathrm{mL}$ in $0.5 \%$ low-viscosity CMC (Sigma-Aldrich). Equal volumes of the rifampicin and resveratrol solutions were mixed to obtain the combinatorial medicine containing the two drugs at 1 or $2 \mathrm{mg} / \mathrm{mL}$ each. In some experiments, resveratrol solution was made at $10 \mathrm{mg} / \mathrm{mL} .10 \mu \mathrm{L}$ of rifampicin (i.e., $0.02 \mathrm{mg}$ ), resveratrol ( 0.02 or $0.1 \mathrm{mg})$, combinatorial medicine $(0.01$ or $0.02 \mathrm{mg}$ each drug), or CMC solution was administered into the bilateral nasal cavity using microtips 5 days a week from Monday to Friday over 1 month. Age-matched non-Tg littermates were treated with CMC alone.

\section{Behavioral Tests}

Following 1-month drug treatment, the cognitive function of the mice was evaluated by the Morris water maze test as described previously (Umeda et al., 2015, 2021). Mice were trained to swim to a hidden platform 5 times a day at 5-min intervals over 4 consecutive days. The time when mice climbed on the platform was recorded. The mean time of the 5 trials was calculated each day. Drug treatment was continued during the behavioral test.
At day 5, the retention of spatial reference memory was assessed by a probe trial consisting of a $60 \mathrm{~s}$ free swim in the pool without the platform.

\section{Hepatotoxicity Assay}

Hepatotoxicity of the drugs was assessed by measuring the plasma levels of liver enzymes. After the water maze task,

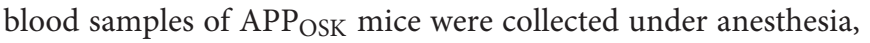
and the plasma was separated by centrifugation. The levels of aspartate transaminase (AST) and alanine transaminase (ALT) were measured using a 7180 Clinical Analyzer (Hitachi HighTechnologies, Tokyo, Japan) with L-type Wako AST-J2 and ALT-J2 reagents (Wako Pure Chemical Industries, Osaka, Japan).

\section{Immunohistochemical Analysis}

After the water maze task, each group was divided into two groups: one group for immunohistochemical analysis and the other for biochemical analysis. Brain sections of $\mathrm{Tg}$ and non-Tg littermates were prepared as described previously (Tomiyama et al., 2010). To expose the antigens, sections were boiled in $0.01 \mathrm{~N} \mathrm{HCl}, \mathrm{pH} 2$ for $10 \mathrm{~min}$ (for $\mathrm{A} \beta$ oligomers, amyloid plaques, tau oligomers, $\alpha$-synuclein oligomers, and $\mathrm{BDNF}$ ) or $10 \mathrm{mM}$ citrate buffer, $\mathrm{pH} 6$ for $30 \mathrm{~min}$ (for synaptophysin). After blocking with $10 \%$ calf serum overnight, the sections were stained with antibodies for pan-A $\beta(\beta 001)$ (Tomiyama et al., 2010), A $\beta$ oligomers (11A1; IBL, Fujioka, Japan), tau oligomers (TOMA1; Merck-Millipore, Darmstadt, Germany), $\alpha$-synuclein oligomers (Syn33; Merck-Millipore), synaptophysin (SVP-38; Sigma-Aldrich), and BDNF (BDNF\#9; DSHB, Iowa City, IA, United States) essentially as described previously (Tomiyama et al., 2010). The staining was followed by biotin-labeled second antibody (Vector Laboratories, Burlingame, CA, United States), horseradish peroxidase (HRP)conjugated avidin-biotin complex (Vector Laboratories), and a HRP substrate, diaminobenzidine (DAB; Dojindo, Kumamoto, Japan). For synaptophysin, immunoreactivity was visualized with FITC-labeled second antibody (Jackson Laboratory). The stained specimens were viewed under a BZ-X800 fluorescence microscope (Keyence, Osaka, Japan), and the images were collected. $\mathrm{A} \beta$, tau, $\alpha$-synuclein pathologies, synapse loss, and BDNF expression were evaluated by quantifying the staining intensity or area in a constant region in each image using NIH ImageJ software.

\section{Western Blot Analysis of BDNF}

Hippocampal tissues were dissected from the brains and homogenized by sonication in 10 volumes of Tris-buffered saline containing protease inhibitor cocktail (P8340; Sigma-Aldrich). The homogenates were centrifuged at $100,000 \times g$ for $30 \mathrm{~min}$ to separate from insoluble materials. The supernatants were subjected to western blotting with antibodies for BDNF (H-117; Santa Cruz Biotechnology, Dallas, TX, United States) and actin (Sigma-Aldrich) followed by HRP-labeled second antibodies (Bio-Rad Laboratories, Hercules, CA, United States) and a chemiluminescent substrate for HRP (ImmunoStar LD; FujifilmWako, Osaka, Japan). The stained proteins were visualized and 
quantified using an ImageQuant LAS 500 image analyzer (GE Healthcare, Hino, Japan).

\section{Statistical Analysis}

All experiments and data analyses were performed under unblinded conditions. Comparisons of means among more than two groups were performed using ANOVA or twofactor repeated measures ANOVA (for the Morris water maze acquisition test), followed by Fisher's PLSD test. In all the behavioral tests we performed the interactions between group and day were not significant, and thus only the group effects were analyzed. Differences with a $P$-value of $<0.05$ were considered significant.

\section{RESULTS}

To explore more effective and safer medicines than rifampicin alone, we tested the therapeutic effects of rifampicin and resveratrol combination using four kinds of mouse models of neurodegenerative dementia.

The first model we used was $\mathrm{APP}_{\mathrm{OSK}}$ mice, which show $\mathrm{A} \beta$ oligomer accumulation and memory impairment at 8 months but not senile plaques even at 24 months (Tomiyama et al., 2010). Drug solution containing $0.02 \mathrm{mg}$ rifampicin alone, $0.02 \mathrm{mg}$ resveratrol alone, or $0.04 \mathrm{mg}$ combinatorial medicine $(0.02 \mathrm{mg}$ rifampicin $+0.02 \mathrm{mg}$ resveratrol) was intranasally administered every day into 13-month-old mice for 1 month. The spatial reference memory was assessed by the Morris water maze test. Both rifampicin alone and resveratrol alone at $0.02 \mathrm{mg} / \mathrm{day}$ appeared to affect mouse memory, but their effects were not significant (Figure 1A). In contrast, the combination of the two drugs showed a significant improvement of memory, as indicated by the test performance nearing that of non- $\mathrm{Tg}$ littermates (Figure 1A). Probe test results appeared to support this conclusion, although no significant differences were detected between groups. We previously reported that nasal rifampicin improved the memory of 11-month-old $\mathrm{APP}_{\mathrm{OSK}}$ mice almost completely at $0.05 \mathrm{mg} /$ day (Umeda et al., 2018). The present results suggest that the combinatorial medicine achieved a sufficient recovery of mouse memory even at a lower dose of rifampicin than rifampicin used alone. This observation implies that by combining with resveratrol, we can lower the risk of rifampicin's adverse effects without affecting the therapeutic efficacy. To further evaluate the therapeutic ability of resveratrol, we tested $0.1 \mathrm{mg}$ resveratrol alone per day at the same time. Resveratrol at this dose showed a significant improvement of memory to a level similar of non-Tg littermates, but the effect was incomplete at days 3 and 4 . Then we evaluated $\mathrm{A} \beta$ oligomer-related pathologies by immunohistochemistry. The levels of $A \beta$ oligomers in the cerebral cortex and hippocampus were significantly attenuated by rifampicin alone and the combinatorial medicine (Figure 1B). Resveratrol alone also diminished the levels, but the effect was weaker even at high dose than that of rifampicin. Rifampicin alone and resveratrol alone at $0.02 \mathrm{mg} /$ day recovered synaptophysin levels in the hippocampal mossy fibers, but again the effects were incomplete (Figure 1C).
In contrast, the combination and high-dose resveratrol restored synaptophysin levels to a level similar of non-Tg littermates. These results imply that mouse memory reflects synaptophysin levels rather than the $\mathrm{A} \beta$ pathology itself.

It has been shown that the levels of BDNF are reduced in the brain of patients with neurodegenerative and psychiatric disorders (Miranda et al., 2019). Resveratrol can induce the expression of BDNF (Shen et al., 2018; Chen et al., 2021). Thus, we examined BDNF levels in brain sections by immunohistochemical staining and in hippocampal tissues by western blot. Immunohistochemistry analysis found that $\mathrm{APP}_{\mathrm{OSK}}$ mice exhibit reduced levels of BDNF in the hippocampal CA2/3 region (Figure 2A). Resveratrol alone restored the levels dosedependently and to a level similar of non- $\mathrm{Tg}$ littermates at $0.1 \mathrm{mg} /$ day. In contrast, rifampicin alone did not significantly affect BDNF levels. The combinatorial medicine recovered BDNF levels similarly to high-dose resveratrol. These results were confirmed by western blots. Hippocampal whole tissues were collected and their soluble fractions were analyzed. The levels of BDNF (14 kD), its precursor pro-BDNF (32 kD), and a truncated form of pro-BDNF (28 kD) (Mowla et al., 2001) were significantly decreased in $\mathrm{APP}_{\mathrm{OSK}}$ mice (Figure $\mathbf{2 B}$ ). Resveratrol alone $(0.02 \mathrm{mg} /$ day $)$ and the combinatorial medicine, but not rifampicin alone, recovered the levels significantly.

To confirm the safety of the combinatorial medicine, we measured the plasma levels of AST and ALT after the behavioral test. Rifampicin-treated mice showed a slight increase in AST levels but almost no change in ALT levels (Table 1). This toxicity by rifampicin was weaker than that reported in our previous study (Umeda et al., 2018), probably because the rifampicin dose in the present study was lower $(0.02 \mathrm{mg} /$ day vs. $0.05 \mathrm{mg} /$ day $)$. In contrast to rifampicin, resveratrol alone did not affect AST levels at $0.02 \mathrm{mg} /$ day but reduced them at $0.1 \mathrm{mg} /$ day. When these two drugs were combined, the rifampicin-induced AST increase vanished, indicating that resveratrol acts on hepatocytes protectively and neutralizes rifampicin's hepatotoxicity, as we expected.

The second model, APP23 mice, which are a typical model of $\mathrm{AD}$, show memory impairment at 3 months and amyloid plaque formation at 6 months (Van Dam et al., 2003). Drug solution containing $0.04 \mathrm{mg}$ combinatorial medicine $(0.02 \mathrm{mg}$ each drug) was intranasally administered every day into 15month-old mice for 1 month. In the Morris water maze, the combination showed a significant improvement of mouse memory to a level similar of non-Tg littermates (Figure 3A). Again, probe test results appeared to support this observation, although the treatment effect was not statistically significant. Immunohistochemical examination revealed that the level of amyloid plaques in the entorhinal cortex was not significantly changed by the treatment, whereas the level of $A \beta$ oligomers was markedly reduced (Figure 3B). These results are consistent with our previous finding that rifampicin reduces $A \beta$ oligomers but not amyloid plaques in Tg2576 mice (Umeda et al., 2016) and imply that not amyloid plaques but $\mathrm{A} \beta$ oligomers are closely associated with cognitive dysfunction in $\mathrm{AD}$. In addition, the combinatorial medicine significantly recovered synaptophysin 

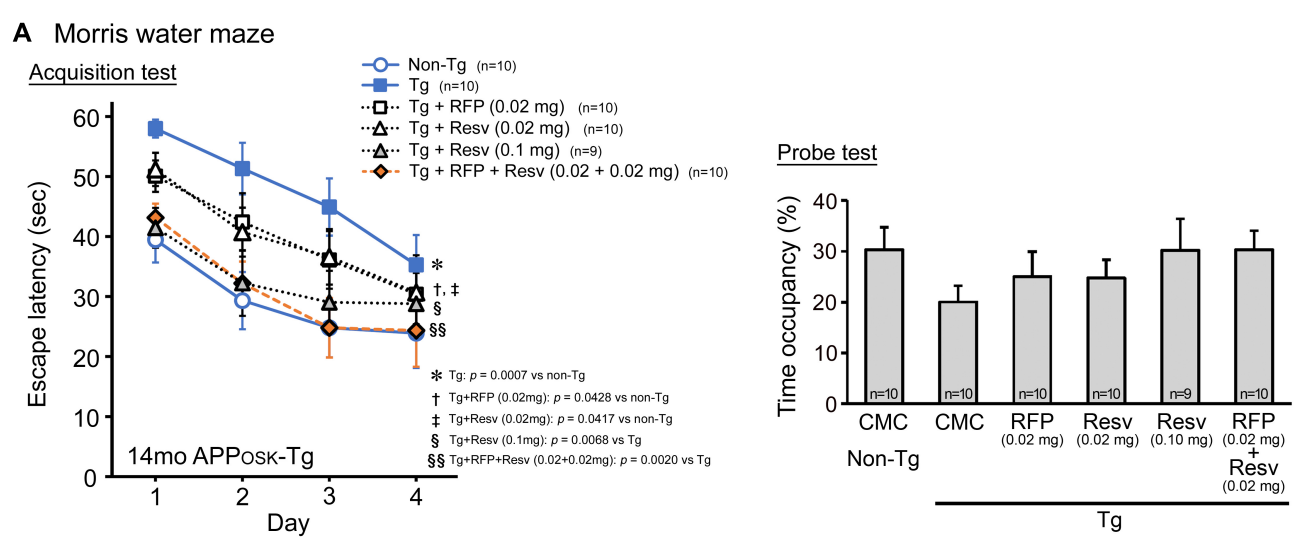

B A $\beta$ oligomers
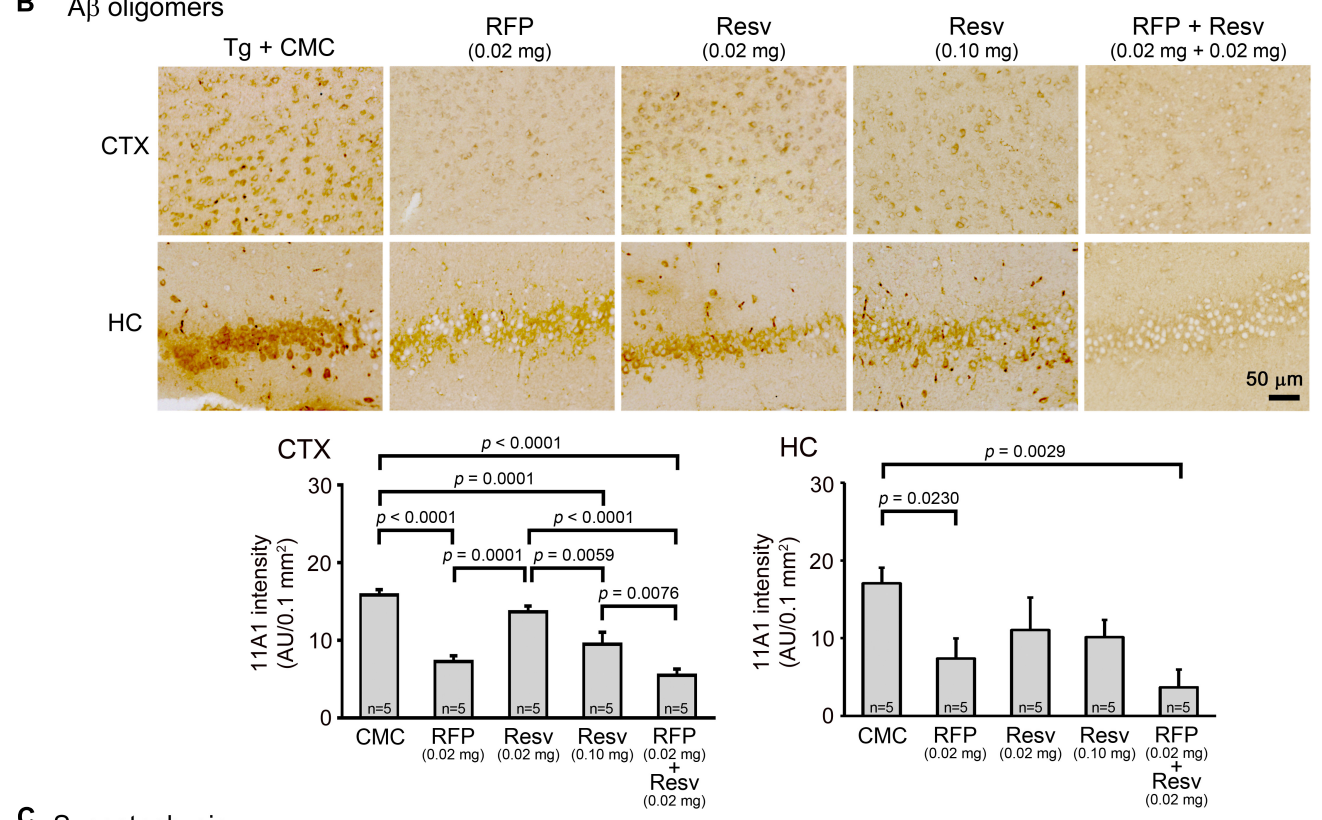

C Synaptophysin
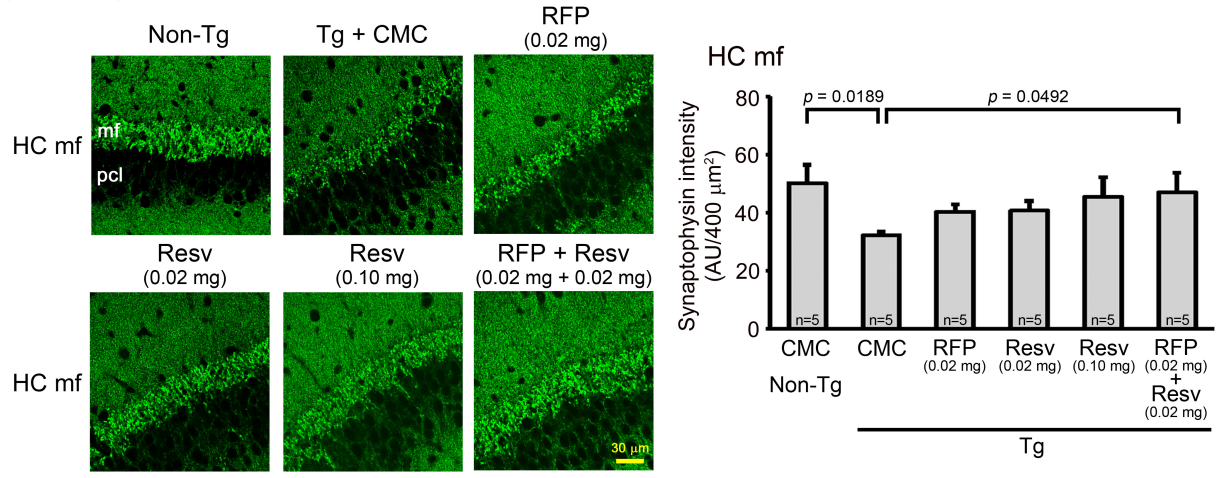

FIGURE 1 | Effects of intranasal rifampicin and resveratrol combination on memory and A oligomer-related pathologies in APPOSK mice. Drug solution containing $0.02 \mathrm{mg}$ rifampicin (RFP) alone, 0.02 or $0.1 \mathrm{mg}$ resveratrol (Resv) alone, $0.04 \mathrm{mg}$ combinatorial medicine (0.02 mg each drug), or carboxymethylcellulose (CMC) as a control was intranasally administered every day into 13-month-old mice for 1 month. (A) In the Morris water maze acquisition test, the combinatorial medicine and high-dose resveratrol showed a significant improvement of mouse memory to a level similar of non-Tg littermates. (B,C) After the water maze task, brain sections were stained with antibodies for $A \beta$ oligomers (11A1) and synaptophysin. (B) The levels of $A \beta$ oligomers in the cerebral cortex (CTX) and hippocampus (HC) were significantly attenuated by rifampicin alone and the combinatorial medicine. (C) The levels of synaptophysin in the hippocampal mossy fibers (HC mf) were significantly recovered by the combinatorial medicine to a level similar of non-Tg littermates. pcl, pyramidal cell layer. AU, arbitrary unit. Values represent the mean \pm SEM. 


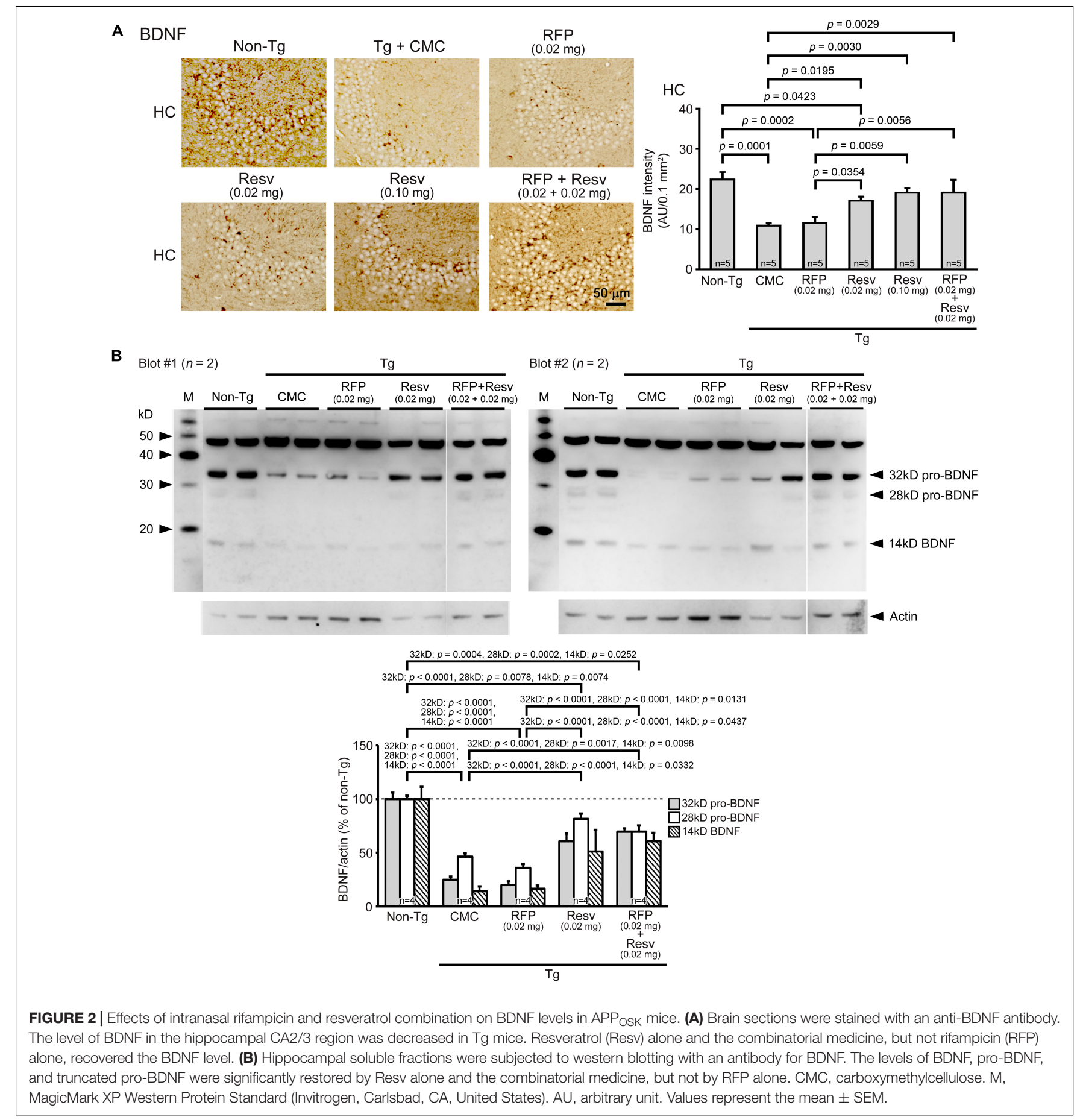

levels in the hippocampal mossy fibers to almost the same level as in non-Tg littermates (Figure 3C).

The third model we used was a model of FTD, tau784 mice, which show tau oligomer accumulation and memory impairment at 6 months (Umeda et al., 2015). Drug solution containing either $0.02 \mathrm{mg}$ rifampicin alone, $0.02 \mathrm{mg}$ resveratrol alone, or $0.04 \mathrm{mg}$ combinatorial medicine $(0.02 \mathrm{mg}$ each drug) was intranasally administered every day into 14-15-month-old mice for 1 month. In the Morris water maze, both rifampicin alone and resveratrol alone at $0.02 \mathrm{mg} /$ day appeared to improve mouse memory, but the effect was significant only in rifampicin (Figure 4A). In contrast, the combination of the two drugs ( $0.02 \mathrm{mg}$ each drug) showed a significant improvement of memory to the same level as non-Tg littermates. We repeated this experiment with a lowdose combinatorial medicine $(0.01 \mathrm{mg}$ each drug). Even at the lower dose, the combination showed a complete recovery of mouse memory. This finding suggests a synergistic effect of the two drugs, although the differences between rifampicin alone 
TABLE 1 | Plasma levels of liver enzymes in APPOSK mice after 1-month intranasal administration of rifampicin, resveratrol, and their combination.

\begin{tabular}{|c|c|c|c|c|c|c|}
\hline & \multirow{2}{*}{$\frac{\text { Non-Tg mice }}{\text { CMC }}$} & \multicolumn{5}{|c|}{ Tg mice } \\
\hline & & CMC & Rifampicin & Resveratrol & Resveratrol & Combination \\
\hline & & & 0.02 mg/day & 0.02 mg/day & $0.1 \mathrm{mg} / \mathrm{day}$ & 0.02 mg each/day \\
\hline & $n=10$ & $n=10$ & $n=10$ & $n=10$ & $n=9$ & $n=10$ \\
\hline AST (IU/L) & $99.5 \pm 12.2$ & $104.4 \pm 8.3$ & $117.6 \pm 15.4$ & $103.7 \pm 9.8$ & $86.0 \pm 12.2$ & $107.7 \pm 18.2$ \\
\hline ALT (IU/L) & $30.9 \pm 3.7$ & $34.1 \pm 2.9$ & $34.4 \pm 3.5$ & $36.9 \pm 4.6$ & $29.1 \pm 12.2$ & $37.2 \pm 4.2$ \\
\hline
\end{tabular}

AST, aspartate transaminase; ALT, alanine transaminase; CMC, carboxymethylcellulose. Values represent the mean \pm SEM.

(0.02 mg) and the higher dose of the combination (0.02 mg each drug) were not significant in both the acquisition and probe tests. Immunohistochemical examination revealed that the levels of tau oligomers in the cerebral cortex, hippocampus, and entorhinal cortex were significantly reduced by rifampicin alone and the low-dose combinatorial medicine (Figure 4B). Resveratrol alone also attenuated the levels, but the effects were significant only in the hippocampus and entorhinal cortex. Images revealed that the effects of rifampicin alone and resveratrol alone on tau oligomers appeared incomplete, whereas that of the combination was almost complete. This observation supports the synergistic effect in the behavioral test. Our results in Figures 1B, 4B collectively indicate that the anti-oligomer activity of rifampicin is stronger than that of resveratrol. Finally, the level of synaptophysin in the hippocampal mossy fibers was recovered by these treatments (Figure 4C). Again, the effects of rifampicin alone and resveratrol alone were not significant, but the level with the combinatorial medicine $(0.02 \mathrm{mg} /$ day) was similar to that of non-Tg littermates.

The fourth and last model was $\alpha$ Syn-Tg mice, which show $\alpha$-synuclein oligomer accumulation in the hippocampus and memory impairment at 6 months, but not motor dysfunction until 9 months (Umeda et al., 2021). We considered this mouse line at 6-8 months a model of DLB. Drug solutions containing 0.02 or $0.04 \mathrm{mg}$ combinatorial medicine (0.01 or $0.02 \mathrm{mg}$ each drug) were intranasally administered every day into 7-month-old mice for 1 month. In the Morris water maze, the combinatorial medicine showed a significant improvement of memory, but only the higher dose caused an improvement that reached a level similar of non-Tg littermates (Figure 5A). In our previous report, nasal rifampicin significantly improved mouse memory when administered to 6-month-old $\alpha$ Syn-Tg mice at $0.1 \mathrm{mg} /$ day (Umeda et al., 2021). The present results suggest that the combinatorial medicine exhibited a sufficient recovery of mouse memory at a lower dose than rifampicin alone. Immunohistochemical examination revealed that the levels of $\alpha$-synuclein oligomers in the visual cortex and hippocampus were significantly reduced by the treatments (Figure 5B). Furthermore, the levels of synaptophysin in the hippocampal mossy fibers was recovered by the treatments (Figure 5C). For both $\alpha$-synuclein oligomers and synaptophysin, however, sufficient amelioration required the high dose of combination.

Taken together, these results indicate that the combination of rifampicin and resveratrol has therapeutic potential in the prevention of neurodegenerative dementia with several advantages over single-drug rifampicin in terms of safety, effectiveness, and enhanced BDNF expression.

\section{DISCUSSION}

Based on recent findings from clinical trials, a consensus has been established that the treatment of neurodegenerative dementia should be started earlier from the asymptomatic, prodromal stages, which are before neurons die. This means that prevention is important for the treatment of these diseases. Here, we propose five requirements of preventive medicines for neurodegenerative dementia. First, preventive medicines must be extremely safe. In prevention, drug treatment is started from the middle to old ages and lasts throughout the lifetime. Notably, several anti$\mathrm{A} \beta$ vaccines and antibodies have been terminated due to their adverse events despite their success at removing senile plaques (Nicoll et al., 2019; Avgerinos et al., 2021). Second, their cost should be reasonable. Particularly in emerging countries, growing medical expenses due to increasingly aging populations are a serious problem. Third, they should be easily administered so that patients can take the drug by themselves at home without the help of medical professionals. Because preventive medicines are to be used repeatedly for many years, they should be provided in a form suitable for self-medication. Fourth, they should have a broad spectrum against amyloidogenic protein oligomers, because we cannot predict which neurodegenerative diseases we will suffer from in the future. Therefore, the drugs should act on not only $\mathrm{A} \beta$ but also tau and $\alpha$-synuclein. Fifth, they must enter the brain and cells efficiently. Tau and $\alpha$-synuclein, and in some cases $\mathrm{A} \beta$, accumulate within neurons and/or glial cells to form toxic oligomers. Efficient brain and cell entry will provide sufficient clinical effects at low dose, low risk, and low cost of the drug.

To fulfill these requirements, in the present study, we combined two neuroprotective compounds, rifampicin and resveratrol, and chose the nose-to-brain route for their administration. Both drugs have been shown to have antioxidant, anti-inflammatory, and anti-amyloidogenic actions (Tomiyama et al., 1996; Bi et al., 2011; Umeda et al., 2016; Ashrafizadeh et al., 2020; Meng et al., 2020; Rahman et al., 2020), but several problems have been observed when either is used alone. The intranasal combination of the two drugs can overcome these problems and even shows several advantages over single-drug treatment. First, regarding safety, rifampicin is an antibiotic whose adverse effects are well recognized. It occasionally induces 


\section{A Morris water maze}

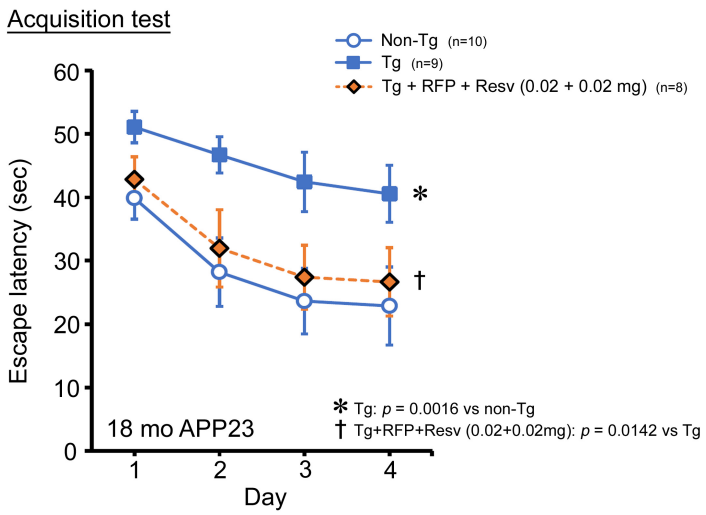

$\underline{\text { Probe test }}$

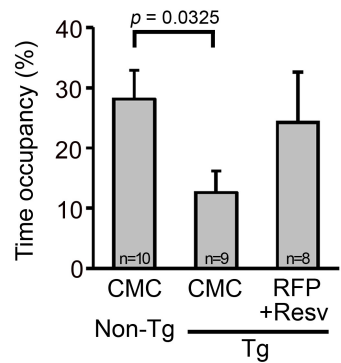

B $\quad A \beta$ pathology

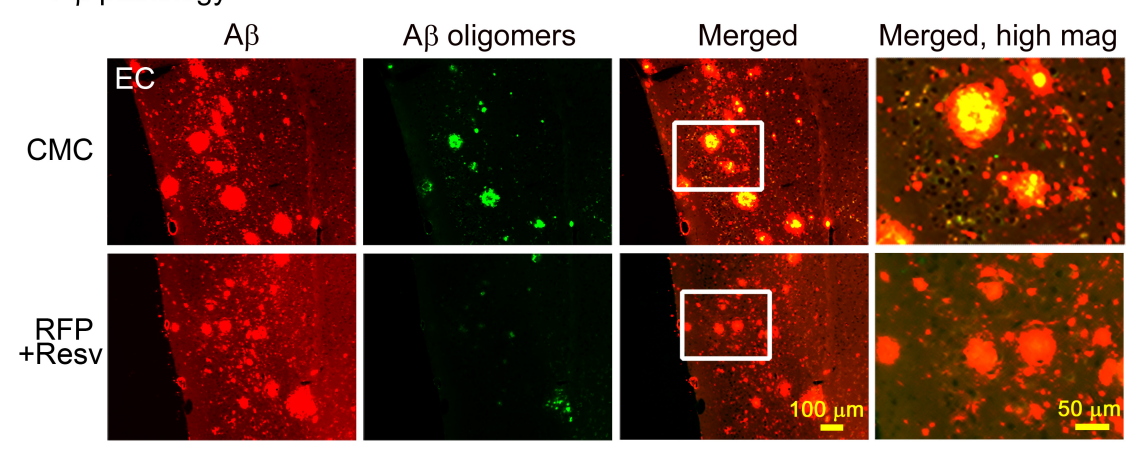

C Synaptophysin

$\mathrm{HC} \mathrm{mf}$
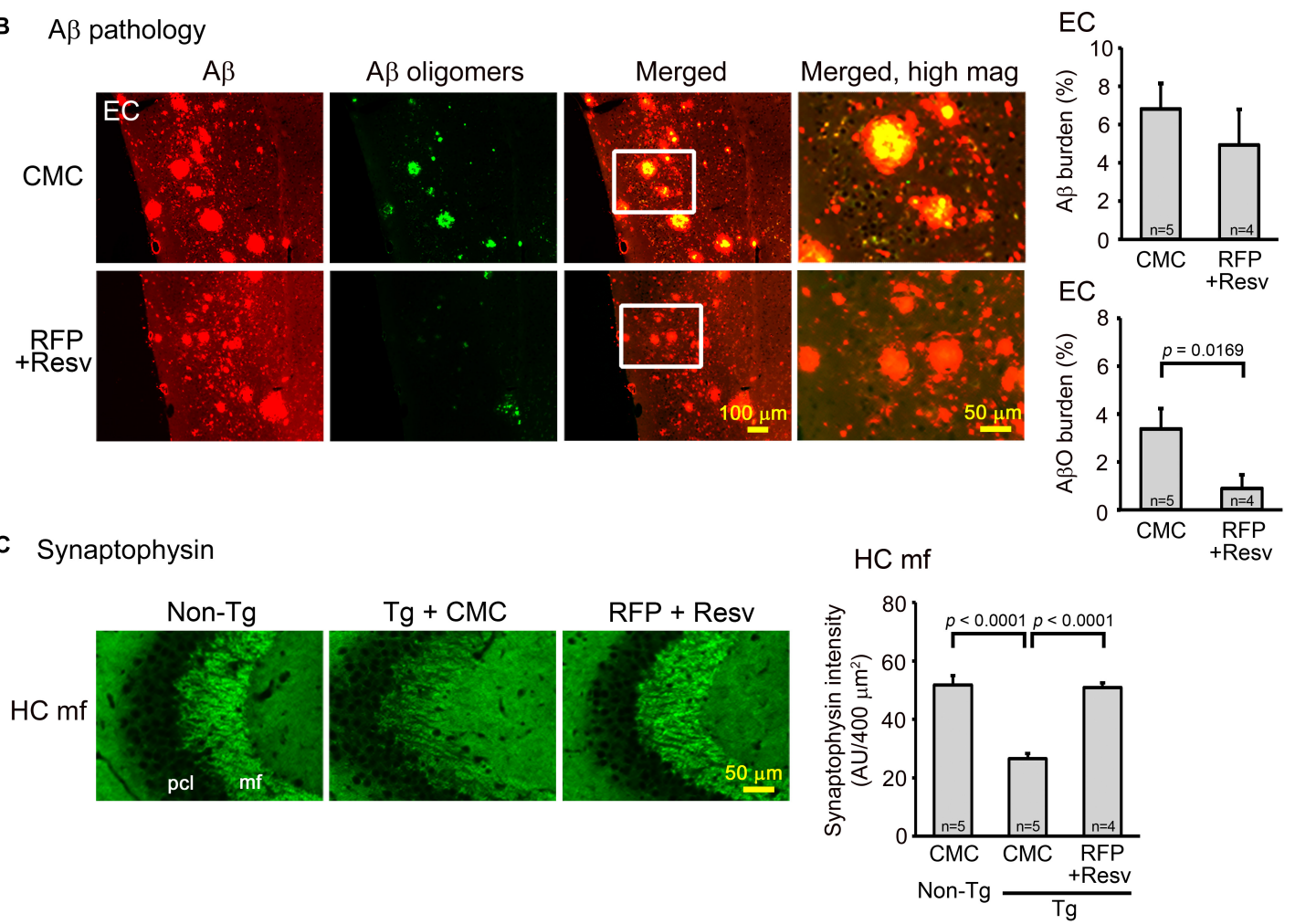

FIGURE 3 | Effects of intranasal rifampicin and resveratrol combination on memory and A $\beta$ pathologies in APP23 mice. Drug solution containing 0.04 mg combinatorial medicine (0.02 mg each drug) or carboxymethylcellulose (CMC) was intranasally administered every day into 16-17-month-old mice for 1 month.

(A) In the Morris water maze acquisition test, the combinatorial medicine showed a significant improvement of mouse memory to a level similar of non-Tg littermates. (B,C) After the water maze task, brain sections were stained with antibodies for A $\beta$ oligomers (11A1), pan-A $\beta$, and synaptophysin. (B) The level of amyloid plaques in the entorhinal cortex (EC) did not change by the treatment, whereas the level of $A \beta$ oligomers $(A \beta O)$ was significantly reduced. (C) The level of synaptophysin in the hippocampal mossy fibers ( $\mathrm{HC} \mathrm{mf}$ ) was significantly recovered by the treatment to almost the same level as non-Tg littermates. pcl, pyramidal cell layer. AU, arbitrary unit. Values represent the mean \pm SEM.

hepatic injury and drug-drug interaction by inducing CYP3A4 enzyme and P-glycoprotein (Kim et al., 2008; Tan et al., 2016). Resveratrol can antagonize these rifampicin actions by inhibiting the expression of CYP3A4 and P-glycoprotein (Deng et al., 2014; El-Readi et al., 2019). Resveratrol itself is very safe and widely used as a dietary supplement. Second, regarding cost, rifampicin is now an inexpensive generic drug, and resveratrol is also cheap.
Thus, the combinatorial medicine could be provided at low cost. Third, regarding the administration, oral administration is the easiest option. However, oral rifampicin can cause adverse events, and oral resveratrol is easily metabolized into inactive forms (Cottart et al., 2014). Nasal sprays also allow patients to easily and non-invasively take these drugs. Fourth, regarding the spectrum, we previously demonstrated that rifampicin shows a wide range 
A Morris water maze

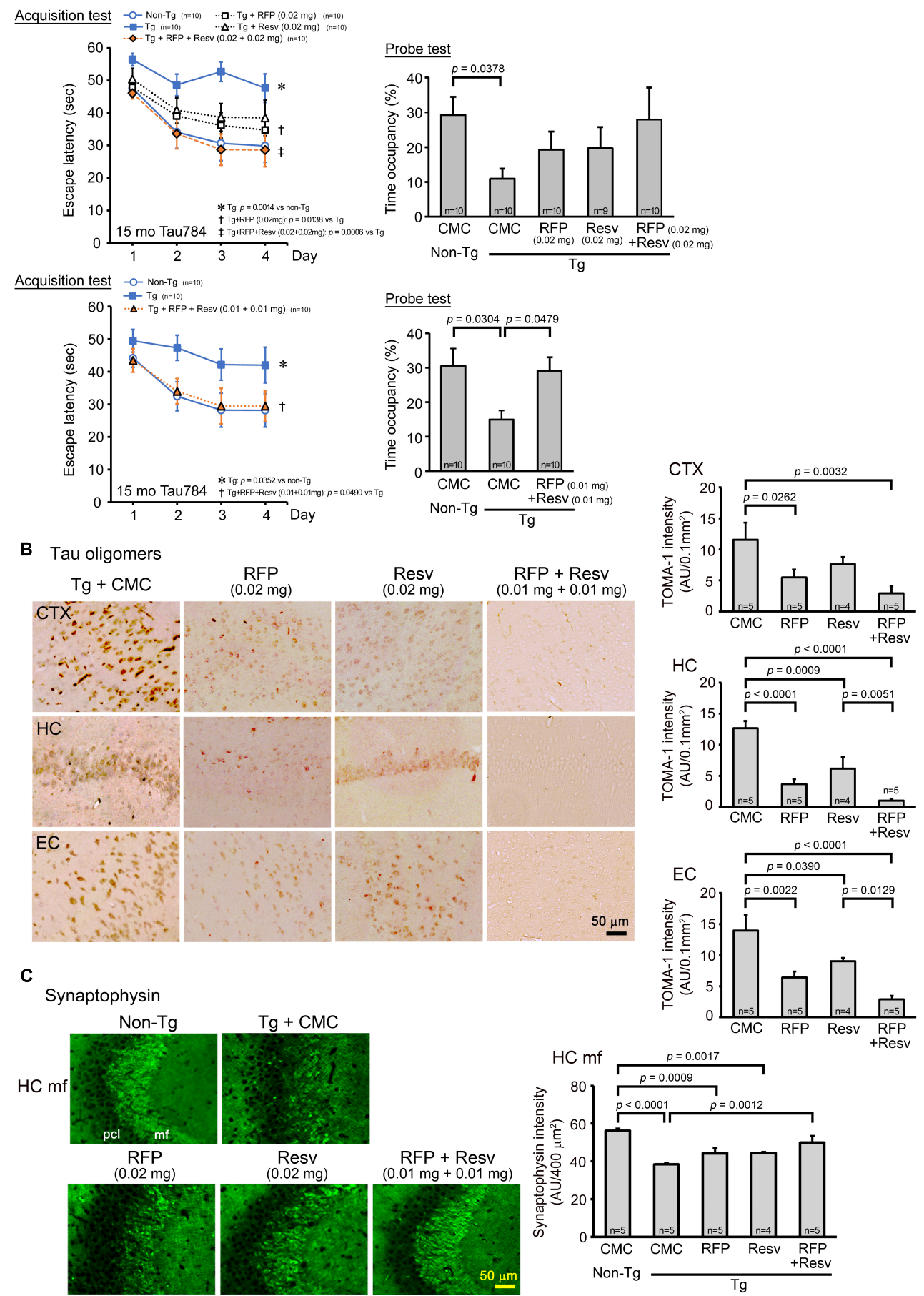

FIGURE 4 | Effects of intranasal rifampicin and resveratrol combination on memory and tau oligomer-related pathologies in tau784 mice. Drug solution containing $0.02 \mathrm{mg}$ rifampicin (RFP) alone, $0.02 \mathrm{mg}$ resveratrol (Resv) alone, 0.02 or $0.04 \mathrm{mg}$ combinatorial medicine (0.01 or $0.02 \mathrm{mg}$ each drug), or carboxymethylcellulose (CMC) was intranasally administered every day into 13-14-month-old mice for 1 month. (A) In the Morris water maze, the combinatorial medicine showed a significant improvement of mouse memory to almost the same level as non-Tg littermates even at the lower dose. This suggests a synergistic effect of rifampicin and resveratrol in ameliorating mouse cognition. (B,C) After the water maze task, brain sections were stained with antibodies for tau oligomers (TOMA-1) and synaptophysin. (B) The levels of tau oligomers in the cerebral cortex (CTX), hippocampus (HC), and entorhinal cortex (EC) were significantly attenuated by rifampicin alone and low-dose $(0.02 \mathrm{mg} /$ day $)$ combinatorial medicine. (C) The level of synaptophysin in the hippocampal mossy fibers (HC mf) was significantly recovered by the combinatorial medicine $(0.02 \mathrm{mg} /$ day $)$ to a level similar of non-Tg littermates. pcl, pyramidal cell layer. AU, arbitrary unit. Values in each figure represent the mean \pm SEM. 


\section{A Morris water maze}
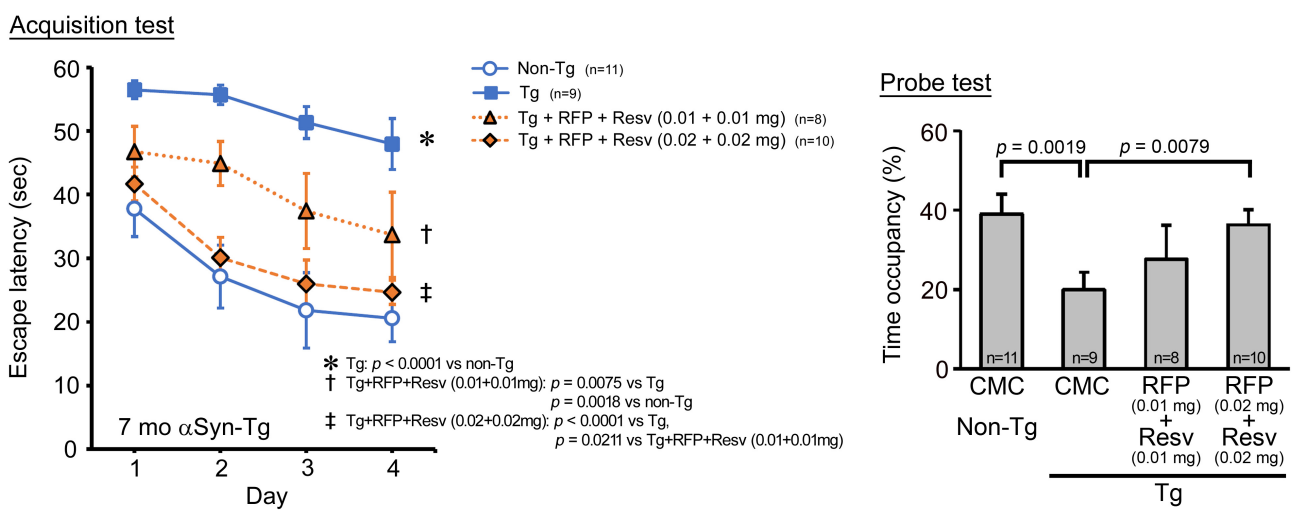

B $\quad \alpha$ Synuclein oligomers
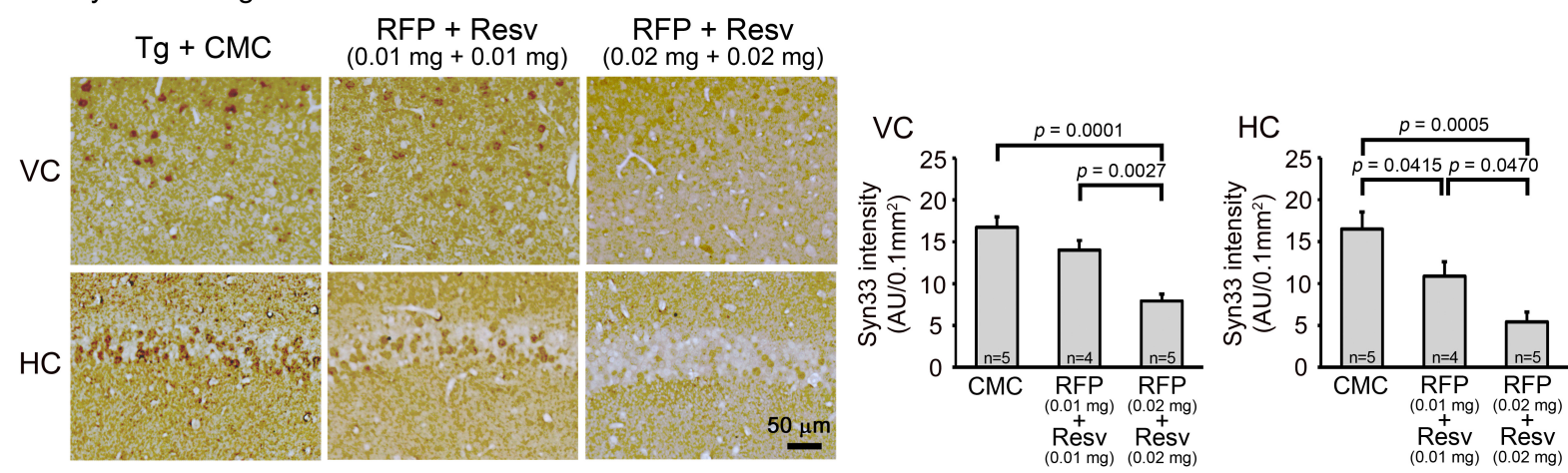

C Synaptophysin
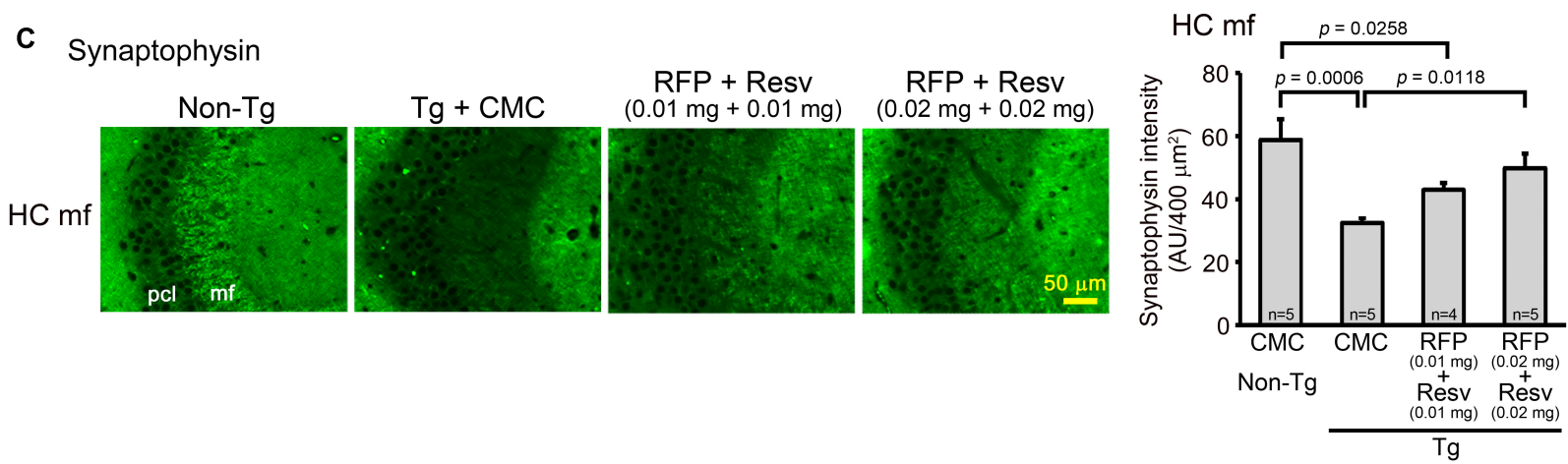

FIGURE 5 | Effects of intranasal rifampicin and resveratrol combination on memory and $\alpha$-synuclein oligomer-related pathologies in $\alpha$ Syn-Tg mice. Drug solution containing 0.02 or $0.04 \mathrm{mg}$ combinatorial medicine $(0.01$ or $0.02 \mathrm{mg}$ each drug) or carboxymethylcellulose (CMC) was intranasally administered every day into 6-month-old mice for 1 month. (A) In the Morris water maze, the combinatorial medicine showed a significant improvement of mouse memory, but to reach a level similar level of non-Tg littermates required the higher dose. (B,C) After the water maze task, brain sections were stained with antibodies for $\alpha$-synuclein oligomers (Syn33) and synaptophysin. (B) The levels of $\alpha$-synuclein oligomers in the visual cortex (VC) and hippocampus (HC) were significantly attenuated by the combinatorial medicine at the higher dose ( $0.04 \mathrm{mg} /$ day). (C) The level of synaptophysin in the hippocampal mossy fibers (HC mf) was significantly recovered by the combinatorial medicine $(0.04 \mathrm{mg} /$ day) to a level similar of non-Tg littermates. pcl, pyramidal cell layer. AU, arbitrary unit. Values in each figure represent the mean $\pm \mathrm{SEM}$.

of anti-oligomer activity and improved cognition of mouse models of AD, FTD, and DLB (Umeda et al., 2016, 2021). In the present study, the combination of rifampicin and resveratrol also rescued cognition and oligomer-related pathologies in these mouse models. Fifth and last, regarding bioavailability in the brain, we showed that the intranasal administration of rifampicin enhanced its brain delivery (Umeda et al., 2018). Furthermore, the intracellular accumulation of tau and $\alpha$-synuclein oligomers, as well as $\mathrm{A} \beta$ oligomers in $\mathrm{APP}_{\mathrm{OSK}}$ mice, were cleared by the intranasal administration of rifampicin, resveratrol, and their combination. These results suggest that intranasal rifampicin and resveratrol can penetrate the brain and cells.

It is noteworthy that by combining rifampicin and resveratrol, more than an additive effect was observed in ameliorating mouse 
cognition and tau pathologies, although memory retention was not significantly rescued by the treatment. This finding implies that the combination can reduce the rifampicin dose to lower the risk of adverse events while keeping the therapeutic effects. One possible explanation of the positive interaction in their effects is that resveratrol has several actions that rifampicin does not have. For example, resveratrol induces the expression of BDNF, probably through an NAD-dependent deacetylase sirtuin1 (SIRT1) signaling pathway (Shen et al., 2018; Chen et al., 2021). BDNF plays an important role in synaptic formation and contributes to memory function (Miranda et al., 2019). We confirmed here that resveratrol alone and the combination increased BDNF and pro-BDNF levels in mouse hippocampi, but rifampicin alone did not. In addition, resveratrol increases insulin sensitivity by activating SIRT1 and AMP-activated protein kinase (AMPK) (Dludla et al., 2020; Meng et al., 2020). Insulin has been shown to function in synaptic remodeling and neurogenesis (Mainardi et al., 2015) and protects neurons from A $\beta$ oligomers (De Felice et al., 2009). Insulin resistance and type 2 diabetes are suggested as risk factors for $\mathrm{AD}$ (Wei et al., 2021). Intranasal insulin is now undergoing clinical trials in $\mathrm{AD}$, mild cognitive impairment, $\mathrm{PD}$, and multiple system atrophy (Alzforum website ${ }^{2}$ ). Although we have not examined the effects of resveratrol on insulin sensitivity in our mice, these features of resveratrol, at least the effect on BDNF, would compensate for rifampicin's inability to repair injured neurons. Furthermore, resveratrol can induce autophagy via several signaling pathways, such as the mammalian target of rapamycin (mTOR), SIRT1, the PI3K/protein kinase B pathway (Akt), and mitogen-associated protein kinase (MAPK) (Pineda-Ramírez and Aguilera, 2021). Autophagy is a cellular waste clearance system both for the generation of materials and removal of toxic components, and its dysregulation has been observed in several neurodegenerative diseases (Malik et al., 2019). We previously reported that rifampicin also restores autophagy in mice (Umeda et al., 2016), but its mechanism likely reduced the burden on autophagy and seems to be different from that of resveratrol. This may explain the much strong effect of the combinatorial medicine in attenuating tau pathologies. The anti-oligomer actions of rifampicin and neurotrophic actions of resveratrol could synergistically function to improve mouse cognition.

Following our earlier reports (Tomiyama et al., 1994, 1996, 1997), a clinical trial with oral rifampicin and doxycycline was performed for mild to moderate AD. Unfortunately, no beneficial effects on cognition or function were observed (Molloy et al., 2013). Clinical trials with oral resveratrol were also carried out for mild to moderate $\mathrm{AD}$, but again no significant clinical outcomes were observed (Turner et al., 2015; Zhu et al., 2018). These results do not necessarily indicate the ineffectiveness of either drug. We hypothesize the main reason for these failures is the late timing of the medication. A recent retrospective study in nondemented patients treated with rifampicin for mycobacterium infections indicated that oral rifampicin is effective at preventing $\mathrm{AD}$, but this effect needs at least $450 \mathrm{mg}$ daily for 1 year (Iizuka et al., 2017). If the treatment is started before the

${ }^{2}$ https://www.alzforum.org/therapeutics/nasal-insulin neurodegeneration and the drug is administered intranasally, rifampicin and resveratrol might be effective at prevention AD. Regarding the daily dose, our results show that at least $0.02 \mathrm{mg}$ rifampicin and $0.02 \mathrm{mg}$ resveratrol are necessary for sufficient cognitive improvement in APP23 and $\alpha$ Syn-Tg mice. Assuming a mouse body weight of $20 \mathrm{~g}$, the $0.02 \mathrm{mg} /$ day dose corresponds to $1 \mathrm{mg} / \mathrm{kg}$ a day. When we calculate the human equivalent dose based on the body surface area according to Nair and Jacob (2016), a dose of $0.081 \mathrm{mg} / \mathrm{kg}$ a day is needed. This amount is far less than the oral rifampicin daily dose for tuberculosis or leprosy (10 mg/kg a day, maximum $600 \mathrm{mg} /$ day) (Drugs.com website $^{3}$ ). In the present study, we combined rifampicin (MW 822.94) and resveratrol (MW 228.24) at 1:1 in weight and used $\mathrm{CMC}$ as the solvent for the drugs. Further investigation is necessary to determine the appropriate ratio of the two drugs and the adequate solvent for intranasal administration in patients. Pharmacokinetic and general toxicity studies of the intranasally administered drugs are also required. However, since rifampicin and resveratrol are widely used and well-known pharmaceutical and dietary supplements, respectively, we expect that the development of the combinatorial medicine should not be too challenging, which merits their drug repositioning.

In conclusion, the present results indicate that the intranasal administration of rifampicin and resveratrol combination has great advantages over single-drug rifampicin in terms of safety and effectiveness. Our findings provide a feasible means for the prevention of neurodegenerative dementia by targeting toxic oligomers.

\section{DATA AVAILABILITY STATEMENT}

The data obtained in this study are available upon reasonable request to the corresponding author.

\section{ETHICS STATEMENT}

All animal experiments were approved by the Ethics Committee of Osaka City University and performed in accordance with the Guide for Animal Experimentation, Osaka City University.

\section{AUTHOR CONTRIBUTIONS}

TU performed the behavioral test, immunohistochemical staining, statistical analysis, and composed the figures. AS performed the drug administration, immunohistochemical staining, and western blotting. KS contributed to the immunohistochemical staining. AY assisted with the drug administration and the western blotting. TK proposed the drug combination study and provided financial support. TT designed the study, conducted the drug administration, immunohistochemical staining, western blotting, and wrote the manuscript. All authors approved the manuscript.

\footnotetext{
${ }^{3}$ https://www.drugs.com/dosage/rifampin.html
} 


\section{FUNDING}

This study was supported by Medilabo RFP, Inc., Kyoto, Japan, and by the grant from the Research Foundation for Dementia of Osaka, Osaka, Japan.

\section{REFERENCES}

Ashrafizadeh, M., Zarrabi, A., Najafi, M., Samarghandian, S., Mohammadinejad, R., and Ahn, K. S. (2020). Resveratrol targeting tau proteins, amyloid-beta aggregations, and their adverse effects: an updated review. Phytother. Res. 34, 2867-2888. doi: 10.1002/ptr.6732

Avgerinos, K. I., Ferrucci, L., and Kapogiannis, D. (2021). Effects of monoclonal antibodies against amyloid- $\beta$ on clinical and biomarker outcomes and adverse event risks: a systematic review and meta-analysis of phase III RCTs in Alzheimer's disease. Ageing Res. Rev. 68:101339. doi: 10.1016/j.arr.2021.101339

Bengoa-Vergniory, N., Roberts, R. F., Wade-Martins, R., and Alegre-Abarrategui, J. (2017). Alpha-synuclein oligomers: a new hope. Acta Neuropathol. 134, 819-838. doi: 10.1007/s00401-017-1755-12

Bi, W., Zhu, L., Wang, C., Liang, Y., Liu, J., Shi, Q., et al. (2011). Rifampicin inhibits microglial inflammation and improves neuron survival against inflammation. Brain Res. 1395, 12-20. doi: 10.1016/j.brainres.2011.04.0190

Chen, J. J., Shen, J. X., Yu, Z. H., Pan, C., Han, F., Zhu, X. L., et al. (2021). The antidepressant effects of resveratrol are accompanied by the attenuation of dendrite/dendritic spine loss and the upregulation of BDNF/p-cofilin1 levels in chronic restraint mice. Neurochem. Res. 46, 660-674. doi: 10.1007/s11064020-03200-1

Cline, E. N., Bicca, M. A., Viola, K. L., and Klein, W. L. (2018). The Amyloid$\beta$ oligomer hypothesis: beginning of the third decade. J Alzheimers Dis. 64, S567-S610. doi: 10.3233/JAD-179941

Cottart, C. H., Nivet-Antoine, V., and Beaudeux, J. L. (2014). Review of recent data on the metabolism, biological effects, and toxicity of resveratrol in humans. Mol. Nutr. Food Res. 58, 7-21. doi: 10.1002/mnfr.201200589

De Felice, F. G., Vieira, M. N., Bomfim, T. R., Decker, H., Velasco, P. T., Lambert, M. P., et al. (2009). Protection of synapses against Alzheimer's-linked toxins: insulin signaling prevents the pathogenic binding of Abeta oligomers. Proc. Natl. Acad. Sci. U S A. 106, 1971-1976. doi: 10.1073/pnas.0809158106

Deng, R., Xu, C., Chen, X., Chen, P., Wang, Y., Zhou, X., et al. (2014). Resveratrol suppresses the inducible expression of CYP3A4 through the pregnane X receptor. J. Pharmacol. Sci. 126, 146-154. doi: 10.1254/jphs.1 $4132 \mathrm{fp}$

Dludla, P. V., Silvestri, S., Orlando, P., Gabuza, K. B., Mazibuko-Mbeje, S. E., Nyambuya, T. M., et al. (2020). Exploring the comparative efficacy of metformin and resveratrol in the management of diabetes-associated complications: a systematic review of preclinical studies. Nutrients 12:739. doi: 10.3390/ nu12030739

El-Readi, M. Z., Eid, S., Abdelghany, A. A., Al-Amoudi, H. S., Efferth, T., and Wink, M. (2019). Resveratrol mediated cancer cell apoptosis, and modulation of multidrug resistance proteins and metabolic enzymes. Phytomedicine 55, 269-281. doi: 10.1016/j.phymed.2018.06.0468

Erdõ, F., Bors, L. A., Farkas, D., Bajza, Á, and Gizurarson, S. (2018). Evaluation of intranasal delivery route of drug administration for brain targeting. Brain Res. Bull. 143, 155-170. doi: 10.1016/j.brainresbull.2018.10.0095

Hill, E., Wall, M. J., Moffat, K. G., and Karikari, T. K. (2020). Understanding the pathophysiological actions of tau oligomers: a critical review of current electrophysiological approaches. Front. Mol. Neurosci. 13:155. doi: 10.3389/ fnmol.2020.00155

Iizuka, T., Morimoto, K., Sasaki, Y., Kameyama, M., Kurashima, A., Hayasaka, K., et al. (2017). Preventive effect of rifampicin on Alzheimer disease needs at least 450 mg daily for 1 Year: an FDG-PET Follow-Up study. Dement Geriatr. Cogn. Dis. Extra 7, 204-214. doi: 10.1159/000477343

Kayed, R., Dettmer, U., and Lesné, S. E. (2020). Soluble endogenous oligomeric $\alpha$-synuclein species in neurodegenerative diseases: expression, spreading, and cross-talk. J. Parkinsons Dis. 10, 791-818. doi: 10.3233/JPD-201965

Kim, K. A., Park, P. W., Liu, K. H., Kim, K. B., Lee, H. J., Shin, J. G., et al. (2008). Effect of rifampin, an inducer of CYP3A and P-glycoprotein, on the

\section{ACKNOWLEDGMENTS}

We thank Momoko Yoshida, Miki Tsutsui, Yukari Hatanaka, Rumi Uekado, Yuki Kinjo, and Yu Masumoto for technical assistance, and Peter Karagiannis for reading the manuscript.

pharmacokinetics of risperidone. J. Clin. Pharmacol. 48, 66-72. doi: 10.1177/ 0091270007309888

Lee, M. K., Stirling, W., Xu, Y., Xu, X., Qui, D., Mandir, A. S., et al. (2002). Human alpha-synuclein-harboring familial Parkinson's disease-linked Ala-53 -> Thr mutation causes neurodegenerative disease with alpha-synuclein aggregation in transgenic mice. Proc. Natl. Acad. Sci. U S A. 99, 8968-8973. doi: 10.1073/pnas. 132197599

Li, S., and Selkoe, D. J. (2020). A mechanistic hypothesis for the impairment of synaptic plasticity by soluble Abeta oligomers from Alzheimer's brain. J. Neurochem. 154, 583-597. doi: 10.1111/jnc.15007

Maeda, S., and Takashima, A. (2019). Tau oligomers. Adv. Exp. Med. Biol. 1184, 373-380. doi: 10.1007/978-981-32-9358-8_27

Mainardi, M., Fusco, S., and Grassi, C. (2015). Modulation of hippocampal neural plasticity by glucose-related signaling. Neural Plast. 2015:657928. doi: 10.1155/ 2015/6579281

Malik, B. R., Maddison, D. C., Smith, G. A., and Peters, O. M. (2019). Autophagic and endo-lysosomal dysfunction in neurodegenerative disease. Mol. Brain 12:100. doi: 10.1186/s13041-019-0504-X

Meng, X., Zhou, J., Zhao, C. N., Gan, R. Y., and Li, H. B. (2020). Health benefits and molecular mechanisms of resveratrol: a narrative review. Foods 9:340. doi: $10.3390 /$ foods 9030340

Miranda, M., Morici, J. F., Zanoni, M. B., and Bekinschtein, P. (2019). BrainDerived neurotrophic factor: a key molecule for memory in the healthy and the pathological brain. Front. Cell Neurosci. 13:363. doi: 10.3389/fncel.2019.00363

Molloy, D. W., Standish, T. I., Zhou, Q., Guyatt, G., Darad Study, and Group. (2013). A multicenter, blinded, randomized, factorial controlled trial of doxycycline and rifampin for treatment of Alzheimer's disease: the DARAD trial. Int. J. Geriatr. Psychiatry 28, 463-470. doi: 10.1002/gps.38461

Mowla, S. J., Farhadi, H. F., Pareek, S., Atwal, J. K., Morris, S. J., Seidah, N. G., et al. (2001). Biosynthesis and post-translational processing of the precursor to brain-derived neurotrophic factor. J. Biol. Chem. 276, 12660-12666. doi: 10.1074/jbc.M0081042000

Nair, A. B., and Jacob, S. (2016). A simple practice guide for dose conversion between animals and human. J. Basic Clin. Pharm. 7, 27-31. doi: 10.4103/09760105.177703

Nicoll, J. A. R., Buckland, G. R., Harrison, C. H., Page, A., Harris, S., Love, S., et al. (2019). Persistent neuropathological effects 14 years following amyloid- $\beta$ immunization in Alzheimer's disease. Brain 142, 2113-2126. doi: 10.1093/brain/ awz142

Pineda-Ramírez, N., and Aguilera, P. (2021). Resveratrol as an inductor of autophagy: is there a unique pathway of activation? Neural Regen. Res. 16, 101-103. doi: 10.4103/1673-5374.286959

Rahman, M. H., Akter, R., Bhattacharya, T., Abdel-Daim, M. M., Alkahtani, S., Arafah, M. W., et al. (2020). Resveratrol and neuroprotection: impact and its therapeutic potential in Alzheimer's disease. Front. Pharmacol. 11:619024. doi: 10.3389/fphar.2020.619024

Shen, J., Xu, L., Qu, C., Sun, H., and Zhang, J. (2018). Resveratrol prevents cognitive deficits induced by chronic unpredictable mild stress: sirt1/miR-134 signalling pathway regulates CREB/BDNF expression in hippocampus in vivo and in vitro. Behav. Brain Res. 349, 1-7. doi: 10.1016/j.bbr.2018.04.0500

Sturchler-Pierrat, C., Abramowski, D., Duke, M., Wiederhold, K. H., Mistl, C., Rothacher, S., et al. (1997). Two amyloid precursor protein transgenic mouse models with Alzheimer disease-like pathology. Proc. Natl. Acad. Sci. U S A. 94, 13287-13292. doi: 10.1073/pnas.94.24.13287

Tan, H., Xu, C., Zeng, H., Wang, Y., Li, Y., Fan, X., et al. (2016). SUMOylation of pregnane $\mathrm{X}$ receptor suppresses rifampicin-induced CYP3A4 and P-gp expression and activity in LS174T cells. J. Pharmacol. Sci. 130, 66-71. doi: 10.1016/j.jphs.2015.11.0062

Tomiyama, T., Asano, S., Suwa, Y., Morita, T., Kataoka, K., Mori, H., et al. (1994). Rifampicin prevents the aggregation and neurotoxicity of amyloid beta protein 
in vitro. Biochem. Biophys. Res. Commun. 204, 76-83. doi: 10.1006/bbrc.1994. 2428

Tomiyama, T., Kaneko, H., Kataoka, Ki, Asano, S., and Endo, N. (1997). Rifampicin inhibits the toxicity of pre-aggregated amyloid peptides by binding to peptide fibrils and preventing amyloid-cell interaction. Biochem. J. 322(Pt 3), 859-865. doi: 10.1042/bj3220859

Tomiyama, T., Matsuyama, S., Iso, H., Umeda, T., Takuma, H., Ohnishi, K., et al. (2010). A mouse model of amyloid beta oligomers: their contribution to synaptic alteration, abnormal tau phosphorylation, glial activation, and neuronal loss in vivo. J. Neurosci. 30, 4845-4856. doi: 10.1523/JNEUROSCI. 5825-09.2010

Tomiyama, T., Shoji, A., Kataoka, K., Suwa, Y., Asano, S., Kaneko, H., et al. (1996). Inhibition of amyloid beta protein aggregation and neurotoxicity by rifampicin. its possible function as a hydroxyl radical scavenger. J. Biol. Chem. 271, 6839-6844. doi: 10.1074/jbc.271.12.6839

Turner, R. S., Thomas, R. G., Craft, S., van Dyck, C. H., Mintzer, J., Reynolds, B. A., et al. (2015). A randomized, double-blind, placebo-controlled trial of resveratrol for Alzheimer disease. Neurology 85, 1383-1391. doi: 10.1212/WNL. 00000000000020351

Umeda, T., Eguchi, H., Kunori, Y., Matsumoto, Y., Taniguchi, T., Mori, H., et al. (2015). Passive immunotherapy of tauopathy targeting pSer413-tau: a pilot study in mice. Ann. Clin. Transl. Neurol. 2, 241-255. doi: 10.1002/acn3.171

Umeda, T., Hatanaka, Y., Sakai, A., and Tomiyama, T. (2021). Nasal rifampicin improves cognition in a mouse model of dementia with lewy bodies by reducing $\alpha$-Synuclein oligomers. Int. J. Mol. Sci. 22:8453. doi: 10.3390/ijms22168453

Umeda, T., Ono, K., Sakai, A., Yamashita, M., Mizuguchi, M., Klein, W. L., et al. (2016). Rifampicin is a candidate preventive medicine against amyloid-beta and tau oligomers. Brain 139(Pt 5), 1568-1586. doi: 10.1093/brain/aww0428

Umeda, T., Tanaka, A., Sakai, A., Yamamoto, A., Sakane, T., and Tomiyama, T. (2018). Intranasal rifampicin for Alzheimer's disease prevention. Alzheimers Dement (N Y) 4, 304-313. doi: 10.1016/j.trci.2018.06.012

Umeda, T., Yamashita, T., Kimura, T., Ohnishi, K., Takuma, H., Ozeki, T., et al. (2013). Neurodegenerative disorder FTDP-17-related tau intron $10+16 \mathrm{C}-\mathrm{T}$ mutation increases tau exon 10 splicing and causes tauopathy in transgenic mice. Am. J. Pathol. 183, 211-225. doi: 10.1016/j.ajpath.2013.03.0153

Van Dam, D., D’Hooge, R., Staufenbiel, M., Van Ginneken, C., Van Meir, F., and De Deyn, P. P. (2003). Age-dependent cognitive decline in the APP23 model precedes amyloid deposition. Eur. J. Neurosci. 17, 388-396. doi: 10.1046/j.14609568.2003.02444.x

Wei, Z., Koya, J., and Reznik, S. E. (2021). Insulin resistance exacerbates Alzheimer disease via multiple mechanisms. Front. Neurosci. 15:687157. doi: 10.3389/ fnins.2021.687157

Zhu, C. W., Grossman, H., Neugroschl, J., Parker, S., Burden, A., Luo, X., et al. (2018). A randomized, double-blind, placebo-controlled trial of resveratrol with glucose and malate (RGM) to slow the progression of Alzheimer's disease: a pilot study. Alzheimers Dement (N Y) 4, 609-616. doi: 10.1016/j.trci.2018.09. 009

Conflict of Interest: TU, TK, and TT have applied for a patent on intranasal rifampicin and resveratrol combination for neurodegenerative dementia (PCT/JP2019/000278). TK and TT are the founders of Medilabo RFP, Inc., who is developing nasal rifampicin. This study received funding from Medilabo RFP, Inc., The funder had the following involvement with the study: TK proposed the drug combination study, and TT designed the study, conducted the drug administration, immunohistochemical staining, western blotting, and wrote the manuscript.

The remaining authors declare that the research was conducted in the absence of any commercial or financial relationships that could be construed as a potential conflict of interest.

Publisher's Note: All claims expressed in this article are solely those of the authors and do not necessarily represent those of their affiliated organizations, or those of the publisher, the editors and the reviewers. Any product that may be evaluated in this article, or claim that may be made by its manufacturer, is not guaranteed or endorsed by the publisher.

Copyright (c) 2021 Umeda, Sakai, Shigemori, Yokota, Kumagai and Tomiyama. This is an open-access article distributed under the terms of the Creative Commons Attribution License (CC BY). The use, distribution or reproduction in other forums is permitted, provided the original author(s) and the copyright owner(s) are credited and that the original publication in this journal is cited, in accordance with accepted academic practice. No use, distribution or reproduction is permitted which does not comply with these terms. 